\title{
Iron Is Filtered by the Kidney and Is Reabsorbed by the Proximal Tubule
}

\author{
Mark Wareing and Craig P. Smith* \\ School of Medical Sciences, The University of Manchester, Manchester, United Kingdom
}

The aim of this study was to determine the iron $(\mathrm{Fe})$ concentration profile within the lumen of the S2 renal proximal convoluted tubule (PCT) and to resolve whether this nephron segment transported Fe. To do this, we performed in vivo renal micropuncture on Wistar rats, collected PCT tubular fluid from superficial nephrons, and measured Fe concentration. The Fe concentration profile along the S2 PCT suggested significant Fe reabsorption. Proximal tubules were also microperfused in vivo with physiological solutions containing

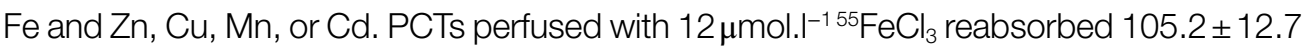
fmol. $\mathrm{mm}^{-1} \cdot \mathrm{min}^{-1} \mathrm{Fe}, 435 \pm 52 \mathrm{pmol} \cdot \mathrm{mm}^{-1} \cdot \mathrm{min}^{-1} \mathrm{Na}$, and $2.7 \pm 0.2 \mathrm{nl} \cdot \mathrm{mm}^{-1} \cdot \mathrm{min}^{-1}$ water

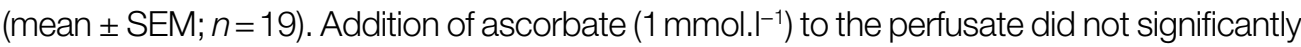
alter $\mathrm{Fe}, \mathrm{Na}$, or water reabsorption. Supplementing the control perfusate with $60 \mu \mathrm{mol} . \mathrm{I}^{-1}$ $\mathrm{FeSO}_{4}$ significantly decreased ${ }^{55} \mathrm{Fe}$ uptake. Recalculating for the altered molar activity following addition of unlabeled Fe revealed a three-fold increase in Fe flux. Addition to the perfusate $12 \mu \mathrm{mol}_{\text {I. }}{ }^{-1} \mathrm{CuSO}_{4}, \mathrm{MnSO}_{4}, \mathrm{CdSO}_{4}$, or $\mathrm{ZnSO}_{4}$ did not affect $\mathrm{Fe}, \mathrm{Na}$, or water

Edited by:

Carolyn Mary Ecelbarger. Georgetown University, United States

Reviewed by: Mykola Mamenko, Augusta University, United States Alan Pao, Stanford University, United States

*Correspondence: Craig P. Smith craig.smith@manchester.ac.uk

Specialty section:

This article was submitted to Renal and Epithelial Physiology,

a section of the journal

Frontiers in Physiology

Received: 13 July 2021 Accepted: 30 August 2021 Published: 30 September 2021

Citation: Wareing M and Smith CP (2021) Iron Is Filtered by the Kidney and is Reabsorbed by the Proximal Tubule. Front. Physiol. 12:740716.

doi: 10.3389/fphys.2021.740716 flux. In conclusion, (1) in vivo, S2 PCTs of rat reabsorb Fe and (2) Fe is reabsorbed along the PCT via a pathway that is insensitive to $\mathrm{Cu}, \mathrm{Mn}, \mathrm{Cd}$, or $\mathrm{Zn}$. Together, these data demonstrate for the first time the hitherto speculated process of renal Fe filtration and subsequent tubular Fe reabsorption in a living mammal.

Keywords: kidney, iron, micropuncture, proximal tubule, glomerular filtration, DMT1, ZIP8, ZIP14

\section{INTRODUCTION}

Iron $(\mathrm{Fe})$ is an essential metal for life. Evolution has harnessed its favorable redox potential, and consequently, $\mathrm{Fe}$ is a key constituent of numerous essential proteins, including hemoglobin, cytochromes, and NADH-coenzyme Q reductase (Ponka, 1999; Williams, 2012). Organisms have evolved mechanisms that exercise fine control over Fe balance because Fe deficiency or excess result in morbidity and mortality. The discovery of Fe transporter proteins expressed in kidney nephron epithelial cells has led to the suggestion that $\mathrm{Fe}$ is reabsorbed by the kidney (Gunshin et al., 1997; Kozyraki et al., 2001; Wolff et al., 2011; Smith et al., 2019). Yet, a key piece of knowledge is lacking in support of this suggestion, namely, there are no direct measures of Fe in kidney tubular fluid or ultrafiltrate. Not surprisingly, in the absence of this fundamental piece of information, the prevailing dogma persists that healthy kidneys do not filter Fe because $\mathrm{Fe}$ is tightly bound to transferrin and in the absence of pathology transferrin is not filtered.

Since the discovery and characterization of the first mammalian Fe transporting protein DMT1 (Gunshin et al., 1997), we have worked to determine the role of this and other Fe 
transporting proteins in the kidney (Smith and Thévenod, 2009; Thévenod and Wolff, 2016). From our work and that of others, it is well established that the components necessary for transcellular Fe transport are expressed in mammalian proximal convoluted tubule (PCT) cells. Briefly, the membranebound divalent metal transporters DMT1, ZIP8, ZIP14, and ferroportin 1 are expressed in PCT cellular membranes (Abouhamed et al., 2006; Wolff et al., 2011; Ajjimaporn et al., 2012; Van Raaij et al., 2018). Furthermore, transferrin receptor 1 and the megalin/cubilin receptor complex, both suggested to mediate reabsorption of filtered protein-bound $\mathrm{Fe}$, are expressed in the apical PCT membrane (Christensen et al., 2012; Smith et al., 2019).

Despite this relative wealth of knowledge, the key questions whether $\mathrm{Fe}$ is present in ultrafiltrate and if so whether $\mathrm{Fe}$ is reabsorbed by the kidney remain unanswered. In view of these gaps in our collective knowledge, the aim of this study was to determine the Fe concentration in PCT S2 rat ultrafiltrate, determine whether the proximal tubule reabsorbs Fe, and begin to characterize the pathway. To do this, we collected nanoliter samples of PCT fluid from anesthetized rats and measured Fe concentration in tubular fluid along the PCT. In addition, using in vivo microperfusion, we measured PCT unidirection flux of radiolabeled $\mathrm{Fe}\left({ }^{55} \mathrm{Fe}\right)$ and assessed the effect of competition by cadmium, copper, manganese, or zinc on ${ }^{55} \mathrm{Fe}$ flux.

\section{MATERIALS AND METHODS}

\section{Study Approval}

All animal studies were performed in accordance with the United Kingdom Animals (Scientific Procedures) Act 1986 and were approved by the University of Manchester Ethics Committee.

\section{Experimental Procedure}

Micropuncture experiments were performed on male Wistar rats $(253 \pm 8 \mathrm{~g} ; n=30)$. Anesthesia was induced with Inactin (5-ethyl-5(1'-methyl-propyl)-2-thiobarbiturate; RBI) at a dose of $100 \mathrm{mgkg}^{-1}$ i.p. Once a satisfactory level of anesthesia was achieved (assessed by the absence of pinch and corneal reflexes), the animal was placed on a thermostatically controlled table set to maintain body temperature at $37^{\circ} \mathrm{C}$. The animal was prepared for micropuncture as previously described (Green et al., 1974). Upon completion of surgery, animals with a proximal tubule transit time in excess of $12 \mathrm{~s}$ or a mean arterial pressure (MAP) below $100 \mathrm{mmHg}$ were rejected from further analysis.

\section{Collection of Tubular Fluid From Proximal Convoluted Tubules Using an Oil Blockade}

Individual PCTs were punctured at random with an oil-filled glass micropipette. An oil block was injected into the tubule lumen, and a timed collection of luminal fluid was made. Two to five collections were made per animal. At the end of an experiment, arterial blood $(5-10 \mathrm{ml})$ was withdrawn via the descending aorta and the animals were subsequently given an overdose of anesthetic. To determine the distance of the collection site from the glomerulus, the collection sites were identified, the tubule lumen was filled with a silicone rubber solution (Microfil; Flow Tek, Boulder, CO), and the filled kidney was removed and stored overnight in deionized water at $4^{\circ} \mathrm{C}$. Casts were then microdissected and measured as previously described (Green et al., 1974).

\section{Microperfusion of Proximal Convoluted Tubules}

Proximal convoluted tubules were perfused at $25 \mathrm{nl} . \mathrm{min}^{-1}$ with physiological solutions using a continuous microperfusion technique (see Bank and Aynedjian, 1972). The control perfusate

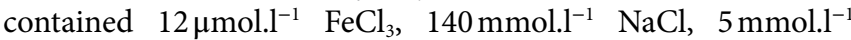

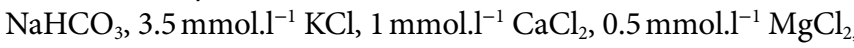
$0.05 \%$ erioglaucine dye, ${ }^{14} \mathrm{C}$-inulin at $12.5 \mu \mathrm{Ci} \cdot \mathrm{ml}^{-1}$ (specific activity $=4.42 \mathrm{mCi} . \mathrm{g}^{-1}$; ICN, Basingstoke, United Kingdom), and $50 \mu \mathrm{Ci} . \mathrm{ml}^{-155} \mathrm{FeCl}_{3}$ (specific activity $=12.3 \mathrm{mCi} \cdot \mathrm{mg}^{-1}$, Amersham, Bucks, United Kingdom) and was gassed to $\mathrm{pH} 6.9,95 \% \mathrm{O}_{2}$, and $5 \% \mathrm{CO}_{2}$. All other experimental groups utilized this solution with the following additions:

Series 1: Control solution plus $1 \mathrm{mmol} . \mathrm{l}^{-1}$ ascorbic acid.

Series 2: Control solution plus $60 \mu \mathrm{mol} . \mathrm{l}^{-1}$ iron sulfate $\left(\mathrm{FeSO}_{4}\right)$.

Series 3: Control solution plus $12 \mu \mathrm{mol}^{-1} \mathrm{l}^{-1}$ either cadmium sulfate $\left(\mathrm{CdSO}_{4}\right)$, copper sulfate $\left(\mathrm{CuSO}_{4}\right)$, manganese sulfate $\left(\mathrm{MnSO}_{4}\right)$, or zinc sulfate $\left(\mathrm{ZnSO}_{4}\right)$.

PCTs with 3-6 surface loops were identified by injection of a small droplet of Sudan Black-stained castor oil into randomly selected tubules. The perfusion pipette was placed downstream of the initial puncture site, and timed collections were made from the sections of the PCT isolated from the rest of the tubule by the injection of mineral oil blocks into the tubule lumen.

At the end of an experiment, arterial blood (5-10 ml) was withdrawn via the descending aorta and the animal euthanized by administering an overdose of anesthetic. The perfused sections of tubules were filled with the silicone rubber solution, and the filled kidney removed and stored overnight in deionized water at $4^{\circ} \mathrm{C}$. The length of the perfused section of tubule was determined from dissection of the silicone rubber casts as previously described (Green et al., 1974).

\section{Measurement of Tubular Fluid, Urine, and Blood Variables}

The collected tubular fluid was dispensed under oil, and the volume was measured from the diameter of the droplet using a calibrated eyepiece micrometer. The concentration of $\mathrm{Fe}$ in the collected fluid was measured using electrothermal atomic absorption spectrophotometer (ETASS; Perkin Elmer Zeeman $3,030)$. Iron concentration was calculated by the standard addition technique (Perkin Elmer, United States). In microperfusion experiments, ${ }^{14} \mathrm{C}$-inulin and ${ }^{55} \mathrm{FeCl}_{3}$ were measured by dual label liquid scintillation counting. The concentration of sodium in the perfusate and collected fluid was measured by ETASS using a previously described protocol (Shalmi et al., 1994). Iron in serum and urine was measured 
by flame atomic absorption spectrophotometry (FAAS; Perkin Elmer 3,100) using previously described tricarboxylic acid (TCA) precipitation protocol (Olson and Hamlin, 1969; Fernandez and Kahn, 1971; Wareing et al., 2000). To measure ultrafiltrable ion concentrations, plasma was spun at $4800 \mathrm{~g}$ RCF for $30 \mathrm{~min}$ through a $30 \mathrm{kDa}$ or $10 \mathrm{kDa}$ filter (Centrifree micropartition system; Amicon). Filtrate was assayed using FAAS. Fluid reabsorptive rate $\left(J_{V}, \mathrm{nl} \cdot \mathrm{mm}^{-1} \cdot \mathrm{min}^{-1}\right)$ was calculated using the following equation:

$$
J_{V}=V_{P}\left(1-I n_{p} / I n_{c}\right) / L
$$

where $V_{p}$ is the tubular perfusion rate $\left(n l \cdot \mathrm{min}^{-1}\right), \mathrm{In}_{\mathrm{p}}$ and $\mathrm{In}_{\mathrm{c}}$ are the concentrations of ${ }^{14} \mathrm{C}$-inulin in perfused and collected fluids, respectively, and $\mathrm{L}$ is the tubule length $(\mathrm{mm})$. Net sodium flux $\left(\mathrm{J}_{\mathrm{Na}}\right)$ was calculated using the following equation:

$$
J_{N a}=V_{p}\left[C_{N a p}-C_{N a c}\left(\operatorname{In}_{p}-\operatorname{In}_{c}\right] / L\right.
$$

where $\mathrm{J}_{\mathrm{Na}}$ is net sodium transport $\left(\mathrm{pmol} \cdot \mathrm{mm}^{-1} \cdot \mathrm{min}^{-1}\right)$, and $\mathrm{C}_{\text {Nap }}$ and $\mathrm{C}_{\mathrm{Nac}}$ are concentrations of sodium (in mmol. $\mathrm{l}^{-1}$ ) in the perfusion solution and the collected fluid, respectively. To calculate the net $\mathrm{Fe}$ flux $\left(\mathrm{J}_{\mathrm{Fe}}\right)$, the specific activity of the radiolabeled $\mathrm{Fe}$ was used to convert the percentage ${ }^{55} \mathrm{Fe}$ flux data to concentration data and these values were then substituted for sodium concentration in the standard equation (Wilson et al., 1997). A positive value for flux indicated reabsorption from the tubule lumen.

Statistical significance for microperfusion experiments was assessed using students $t$ test or single-factor ANOVA using Bonferroni's multiple comparison post hoc test. Values for ion and water fluxes and isotope recoveries are presented as means \pm SEM throughout the text, where $n=$ number of tubules unless stated otherwise.

\section{RESULTS}

\section{Whole Animal Data}

Serum Fe concentration measured using the standard TCA precipitation protocol was $50.5 \pm 3.9 \mu \mathrm{mol}^{-\mathrm{l}^{-1}}(n=23)$. The MAP at the time when collections were made was $103.6 \pm 0.6 \mathrm{mmHg}$ $(n=26)$. The Fe concentration measured in serum that had been spun through micropartition columns was $4.5 \pm 0.4 \mu \mathrm{mol}^{-1}$ $(n=15)$ for the $30 \mathrm{kDa}$ cutoff filter and $2.5 \pm 0.2 \mu \mathrm{mol}^{-1^{-1}}(n=23)$ for the $10 \mathrm{kDa}$ cutoff filter. These values are consistent with our previous measurements and indicated that the serum contained an ultrafilterable component of $\mathrm{Fe}$. Urine $\mathrm{Fe}$ concentration was $4.5 \pm 0.4 \mu \mathrm{mol}^{\mathrm{l}^{-1}}(n=23)$.

\section{Tubular Fluid Collection From Proximal Convoluted Tubules}

The mean tubular fluid collection time was $19.4 \pm 0.7 \min (n=26)$, and the mean volume collected was $249.3 \pm 2.0 \mathrm{nl}(n=26)$. The concentration of $\mathrm{Fe}$ in the collected fluid ranged from 0.9 to $10.5 \mu \mathrm{mol}^{-\mathrm{l}^{-1}}$. The mean Fe concentration in the collected tubular fluid was $3.0 \pm 0.4 \mu \mathrm{mol.} \mathrm{l}^{-1}(n=26)$ and was significantly lower than serum Fe $(p<0.001, n=23)$. Plotting Fe concentration in collected fluid against distance of the collection site from the glomerulus gave a scatter of points (Figure 1). Least squares regression analysis yielded a regression line described by the equation $y=5.026-0.738 \mathrm{x} ; n=26$. However, the correlation coefficient was not significant at the $5 \%$ level $\left(r^{2}=0.13\right)$. Pooling the concentrations of $\mathrm{Fe}$ measured in collections from the initial S2 segment (1-2 mm from the glomerulus) and the collections made in the last S2 segment $(3.5-4.5 \mathrm{~mm}$ from glomerulus) gave means of $3.3 \pm 0.6 \mu \mathrm{mol}^{-l^{-1}}$ and $2.1 \pm 0.5 \mu \mathrm{mol}^{-1}{ }^{-1}$, respectively (Figure 1). Comparing these two means by unpaired $t$ test yielded a $p$ value of 0.13 and deemed the values to be not statistically different at the $p<0.05$ level.

\section{Microperfusion of Proximal Convoluted Tubules}

Recovery of perfused ${ }^{14} \mathrm{C}$-inulin was not significantly different from $100 \%$ for all experimental groups, and therefore, perfusate was not lost during perfusion and differences between the amount of $\mathrm{Fe}$ perfused and recovered must have therefore been due to tubular events. The mean duration of collection, the mean length of the tubules perfused, and the MAP at the time of each collection were not statistically different between groups (Table 1).

Perfusion of PCTs with a physiological solution containing $12 \mu \mathrm{mol}^{-1}{ }^{55} \mathrm{FeCl}_{3}$ (control) resulted in recovery of $66.2 \pm 3.6 \%$ $(n=19)$ of perfused ${ }^{55} \mathrm{Fe}$. This value was significantly different from $100 \%$, and therefore, one-third of Fe we presented to the PCT was reabsorbed. Normalizating the amount of Fe reabsorbed per unit length of tubule gave a value of $105.2 \pm 12.7 \mathrm{fmol} . \mathrm{mm}^{-1}$. $\min ^{-1}(\mathrm{n}=19)$. Plotting $\mathrm{J}_{\mathrm{Fe}}$ against $\mathrm{J}_{\mathrm{v}}$ gave a scatter of points described by the line $y=28.3 x+29.9 \quad(n=19$, Figure 2$)$.



FIGURE 1 | Iron concentration profile along the S2 renal proximal convoluted tubule (PCT). Iron concentration in collection measured by FAAS plotted against distance of collection from glomerulus. Data are plotted for individual tubular fluid collections from 7 animals. Equation of regression line $y=5.026-$ $0.738 x ; n=22 ; r^{2}=0.13$. Bars show mean \pm SEM for collections made in early


S2 (3.5-4.5 mm from glomerulus) $2.1 \pm 0.5 \mu$ mol. $^{-1}, n=7$. Comparison of these two means by unpaired $t$ test yielded a value of $p=0.13$. 
TABLE 1 | Tubular parameters for tubular microperfusion study.

\begin{tabular}{lcrrr}
\hline & $\begin{array}{c}\text { MABP at } \\
\text { time of } \\
\text { collection } \\
\mathbf{( m m H g )}\end{array}$ & $\begin{array}{c}\text { Mean } \\
\text { collection } \\
\text { duration } \\
\text { (mins) }\end{array}$ & \multicolumn{1}{c}{$\begin{array}{c}{ }^{14} \mathbf{C} \text {-inulin } \\
\text { recovery (\%) }\end{array}$} & $\begin{array}{c}\text { Tubule length } \\
(\mathbf{m m})\end{array}$ \\
\hline Control (19) & $103.9 \pm 1.3$ & $3.2 \pm 0.2$ & $99.2 \pm 1.2$ & $1.6 \pm 0.2$ \\
Ascorbate (15) & $101.7 \pm 0.6$ & $3.0 \pm 0.2$ & $99.2 \pm 1.2$ & $1.6 \pm 0.2$ \\
Iron (12) & $101.1 \pm 0.3$ & $3.2 \pm 0.2$ & $100.6 \pm 1.5$ & $2.0 \pm 0.3$ \\
Copper (9) & $105.4 \pm 1.3$ & $2.6 \pm 0.4$ & $100.0 \pm 1.7$ & $1.5 \pm 0.2$ \\
Zinc (7) & $101.3 \pm 0.5$ & $3.3 \pm 0.4$ & $99.7 \pm 1.8$ & $1.8 \pm 0.2$ \\
Manganese (8) & $102.5 \pm 1.21$ & $3.3 \pm 0.4$ & $100.4 \pm 1.7$ & $1.4 \pm 0.1$ \\
Cadmium (9) & $100.9 \pm 0.4$ & $2.9 \pm 0.3$ & $97.2 \pm 1.8$ & $1.5 \pm 0.1$
\end{tabular}

Values are means $\pm S E M$; number of tubules in parentheses; ${ }^{14} \mathrm{C}$-inulin recoveries were not significantly different from $100 \%$. MABP at time of collection, collection duration, and tubule lengths were comparable in each group.

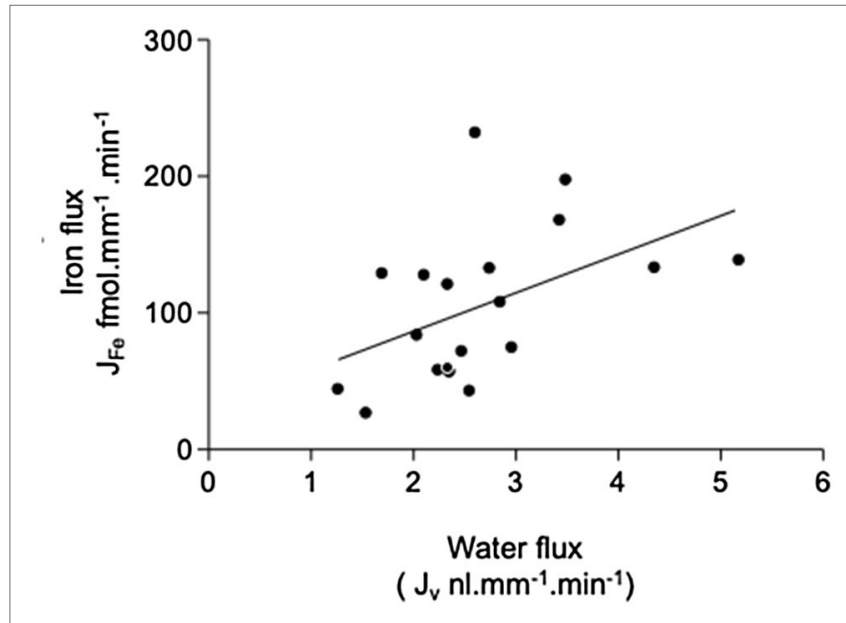

FIGURE 2 | Relationship between iron and fluid reabsorption in the perfused PCT (control data only). Iron flux $J_{F e}$ plotted against water flux $J_{v}$. Data are plotted for individual tubular fluid collections from 5 animals, $n=19$ collections. Equation of regression line $\mathrm{y}=28.26+29.93 \mathrm{x} ; n=19 ; r^{2}=0.23$. The correlation coefficient $r^{2}=0.23$ was significant at the $5 \%$ level, indicating that there was a small significant positive correlation between the reabsorption of iron and water reabsorption.

The correlation coefficient $r^{2}=0.23$ was significant at the $5 \%$ level, and therefore, reabsorption of $\mathrm{Fe}$ was influenced by water reabsorption.

\section{Series 1 \\ Effect of Ascorbate}

Addition of $1 \mathrm{mmol.} \mathrm{l}^{-1}$ ascorbate to the luminal perfusate did not significantly alter the transport of ${ }^{55} \mathrm{Fe}(101.2 \pm 13.0 \mathrm{fmol}$. $\mathrm{mm}^{-1} \cdot \mathrm{min}^{-1}(n=15)$. This treatment produced small increases in sodium reabsorption $(434.9 \pm 52.2 ; \mathrm{n}=19$ to $598 \pm 79 \mathrm{pmol}$. $\left.\mathrm{mm}^{-1} \cdot \mathrm{min}^{-1} ; n=15\right)$ and water transport $(2.7 \pm 0.2 ; n=19$ to $\left.3.2 \pm 0.3 \mathrm{ml} \cdot \mathrm{mm}^{-1} \cdot \mathrm{min}^{-1} ; n=15\right)$. These increases were not statistically significant at the $5 \%$ level (t test, $p=0.08$ and 0.16, respectively; Figure 3). Because ascorbate had no effect on PCT Fe reabsorption, it was not included in further experiments.

\section{Series 2 \\ Effect of Fe Sulfate $\left(\mathrm{FeSO}_{4}\right)$}

Addition of $60 \mu \mathrm{mol} . \mathrm{I}^{-1} \mathrm{FeSO}_{4}$ to the luminal perfusate did not affect sodium reabsorption and caused a small, but not statistically significant, decrease in water reabsorption from $2.7 \pm 0.2$ to $2.1 \pm 0.3 \mathrm{nl} \cdot \mathrm{mm}^{-1} \cdot \mathrm{min}^{-1}(n=11$, Figure 4). Transport of ${ }^{55} \mathrm{Fe}$ was reduced from $105.2 \pm 12.7 \mathrm{fmol} . \mathrm{mm}^{-1} \cdot \mathrm{min}^{-1}$ to $53.5 \pm 7.5$ fmol. $\mathrm{mm}^{-1} \cdot \mathrm{min}^{-1}(n=11 ; p<0.01)$. Recalculating the molar activity of ${ }^{55} \mathrm{Fe}$ to take into account the addition of $60 \mu \mathrm{mol} . \mathrm{l}^{-1} \mathrm{Fe}$ revealed that Fe transport as a whole increased to $310.5 \pm 43.4 \mathrm{fmol} \cdot \mathrm{mm}^{-1} \cdot \mathrm{min}^{-1}(n=11 ; p<0.01)$. This showed that the control perfusion of $12 \mu \mathrm{mol}^{-1} \mathrm{Fe}$, although three times higher than the concentration of Fe we measured in the PCT collections, did not saturate the transport pathway.

\section{Series 3 \\ Effect of Copper $\left(\mathrm{CuSO}_{4}\right)$, Zinc $\left(\mathrm{ZnSO}_{4}\right)$, Manganese $\left(\mathrm{MnSO}_{4}\right)$, or Cadmium Sulfate $\left(\mathrm{CdSO}_{4}\right)$} Addition of equimolar $\mathrm{CuSO}_{4}, \mathrm{MnSO}_{4}$, or $\mathrm{ZnSO}_{4}\left(12 \mu \mathrm{mol} . \mathrm{l}^{-1}\right)$ had no significant effect on $\mathrm{Fe}$ reabsorption (Figure 5). This suggested that neither copper, manganese, nor zinc competes with $\mathrm{Fe}$ for transport. $\mathrm{CdSO}_{4}$ caused marginal decreases in water and sodium reabsorption $(p=0.25$ and 0.15 , respectively; ANOVA), but Fe absorption was unaffected by these reductions in transport.

\section{DISCUSSION}

The aim of the current study was to determine the $\mathrm{Fe}$ concentration in ultrafiltrate collected from the lumen of rodent S2 proximal tubule and to determine whether Fe is reabsorbed by the PCT. To do this, we utilized in vivo renal micropuncture and measured $\mathrm{Fe}$ concentration in samples of ultrafiltrate collected from S2 nephron segment. We also performed divalent metal competition studies to begin to functionally characterize the $\mathrm{Fe}$ reabsorptive pathway.

Tubular fluid collected from rat PCT contained Fe. This finding suggests $\mathrm{Fe}$ is either filtered by the glomerulus or secreted into the tubular lumen. By comparing tubular $\mathrm{Fe}$ concentration to the distance, a collection was made from the glomerulus and we determined whether Fe was reabsorbed or secreted. Because $50-60 \%$ of the water in the ultrafiltrate is reabsorbed from the PCT, a solute that is not reabsorbed or one that is secreted would increase in concentration the further a collection was made from the glomerulus (Rector, 1983). In the case of isosmotic reabsorption, no change in concentration would be expected and for higher rates of reabsorption the concentration of an ion/solute along the PCT would decrease. By comparing collections made in the first and last accessible millimeter of the S2 PCT, it was very evident that there was no discernable increase in PCT $\mathrm{Fe}$ and the concentration 


\section{A}

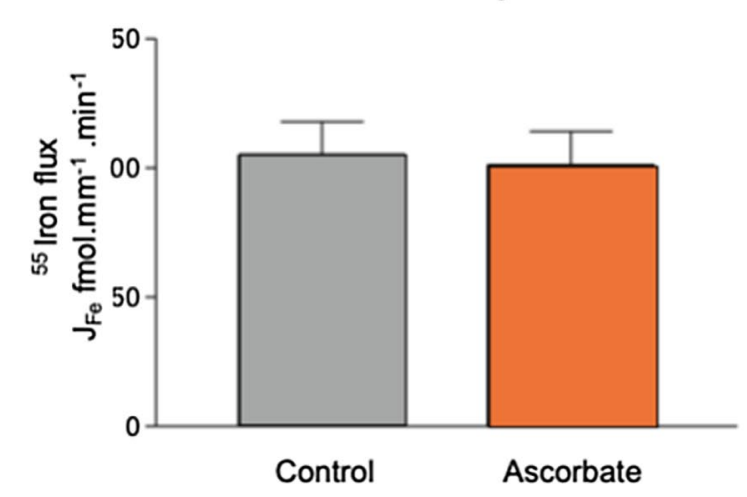

B

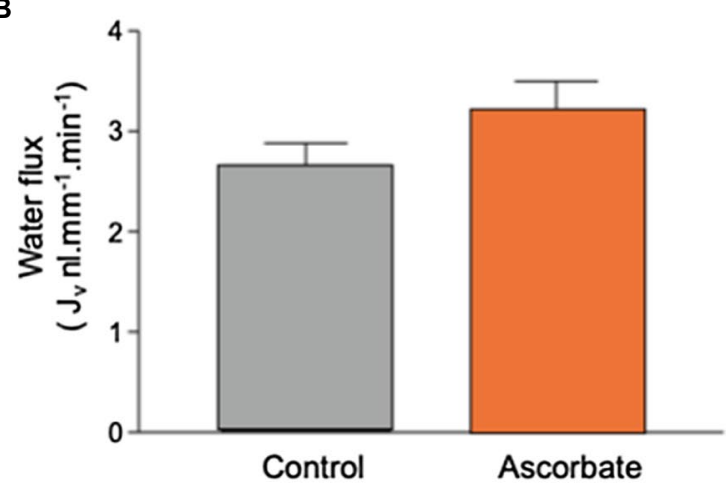

C

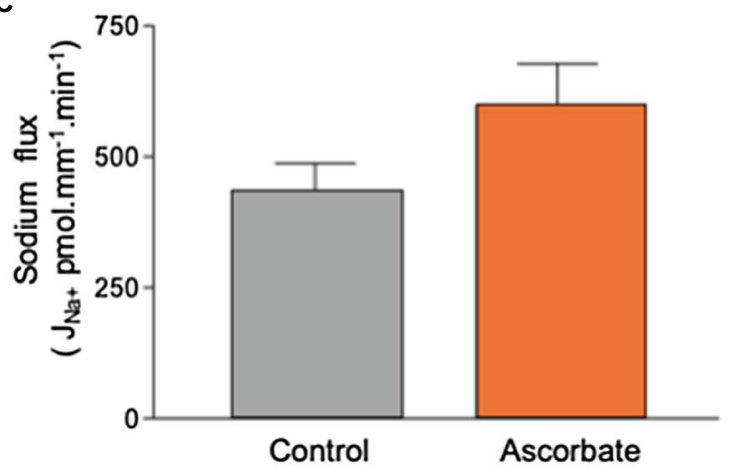

FIGURE 3 | Effect of $1 \mathrm{mmol} . \mathrm{I}^{-1}$ ascorbate on ${ }^{55} \mathrm{Fe}$, water, and $\mathrm{Na}^{+}$flux in microperfused renal PCT. Surface S2 PCTs were microperfused in vivo, and the effect of $1 \mathrm{mmol} . \mathrm{I}^{-1}$ ascorbic acid on ${ }^{55} \mathrm{Fe}$ flux determined. Control perfusate contained $12 \mu \mathrm{mol} . \mathrm{I}^{-155} \mathrm{FeCl}_{3}$. Ascorbate perfusate consisted of control perfusate plus $1 \mathrm{mmol} . \mathrm{I}^{-1}$ ascorbic acid (A) Mean Fe flux of $105.2 \pm 12.7 \mathrm{fmol} \cdot \mathrm{mm}^{-1} \cdot \mathrm{min}^{-1}(n=19)$ was measured. Addition of $1 \mathrm{mmol} . \mathrm{I}^{-1}$ ascorbic acid resulted in a mean Fe flux of $101.2 \pm 13.0 \mathrm{fmol} . \mathrm{mm}^{-1} \cdot \mathrm{min}^{-1}$ $(n=15)$. (B) Water flux by microperfused PCT was not significantly affected by the addition of ascorbic acid $\left(2.7 \pm 0.2 ; n=19\right.$ to $3.2 \pm 0.3 \mathrm{nl} \cdot \mathrm{mm}^{-1} \cdot \mathrm{min}^{-1}$; $n=15)$. (C) Sodium flux was slightly elevated by inclusion of ascorbate, but the difference did not reach statistical significance at $0.5 \%$ level $(434.9 \pm 52.2$; $n=19$ to $\left.598 \pm 79 \mathrm{pmol} \cdot \mathrm{mm}^{-1} \cdot \mathrm{min}^{-1} ; n=15\right)$.

remained relatively constant or if anything decreased slightly along the PCT. These findings when taken together strongly suggest $\mathrm{Fe}$ is not secreted into the tubule lumen, but that it is filtered by the glomerulus and reabsorbed along the proximal tubule.

Comparing Fe flux and water flux enabled us to determine whether reabsorption of Fe might be linked to water movement. We found a correlation between water and Fe flux, indicating that the two processes were linked. This was not unexpected given that movement of a solute across a membrane generates an osmotic gradient. On the other hand, movement of water out of the tubule serves to increase the effective concentration of a solute and hence increases the solute concentration gradient thus favoring transport out of the tubular lumen. It was not possible from our experiments to definitively determine which mechanism led to the observed coupled movement; however, the finding that PCT Fe and water movement were linked reinforces the conclusion that $\mathrm{Fe}$ is reabsorbed from the PCT.

To begin to functionally characterize the proposed reabsorptive pathway, we performed a series of PCT microperfusion experiments. We perfused PCTs and measured the flux of ${ }^{55} \mathrm{Fe}$ out of the proximal tubule as a marker for Fe reabsorption. To ensure that the PCT was functioning normally, we simultaneously measured sodium and water flux and obtained values very comparable to those previously reported by our laboratory under similar perfusion conditions (Wareing and Green, 1994; Wareing et al., 1995). The mean recovery of $\mathrm{Fe}$ from PCT's perfused with a solution containing $12 \mu \mathrm{mol} . \mathrm{l}^{-1}$ Fe was $66 \%$. Therefore, on average, a third of the Fe presented to the PCT was taken up by the tubule. The rate of Fe transport measured was $105.2 \pm 12.7 \mathrm{fmol} . \mathrm{mm}^{-1} \cdot \mathrm{min}^{-1}$, and since this is the first measurement of PCT Fe flux, it is not possible to compare it to other published data, and while the fluxes of other ions out of the PCT (e.g., $\mathrm{Na}, \mathrm{Ca}, \mathrm{K}$, or $\mathrm{Mg}$ ) have been measured by our group and others groups given the methodological differences, it is not possible to directly compare these values.

Adding unlabeled Fe to the perfusate decreased the apparent unidirectional ${ }^{55} \mathrm{Fe}$ flux. This was due to unlabeled Fe competing with ${ }^{55} \mathrm{Fe}$, possibly for a fixed number of transporter carrier binding sites. Recalculating the molar activity of ${ }^{55} \mathrm{Fe}$ to take into account the addition of unlabeled Fe revealed that increasing the Fe concentration five-fold caused a three-fold increase in Fe flux. This outcome is important for two reasons: Firstly, it shows that our experimental protocol was capable of uncovering decreased or increased Fe flux; secondly, it showed that the transport pathway function was concentration dependent.

The membrane-bound metal transporter proteins DMT1, ZIP8, and ZIP14 are expressed in the mammalian PCT (Wang et al., 2012; Van Raaij et al., 2018). These proteins are selective for several divalent metals including $\mathrm{Fe}^{2+}$. With this in mind, our next aim was to determine the characteristics of PCT Fe flux. Firstly, we looked at the effect of including ascorbate in the perfusate, reasoning that DMT1, ZIP8, and ZIP14 mediate the transport of $\mathrm{Fe}^{2+}$ and the perfusate we used contained predominately $\mathrm{Fe}^{3+}$; thus, inclusion of ascorbate would be expected to increase Fe flux (Sparkman et al., 2011; Illing et al., 2012; Wang et al., 2012). We found ascorbate did not alter Fe flux suggesting that ascorbate was not necessary for PCT Fe translocation. Ferrous $\mathrm{Fe}^{2+}$ is promoted 


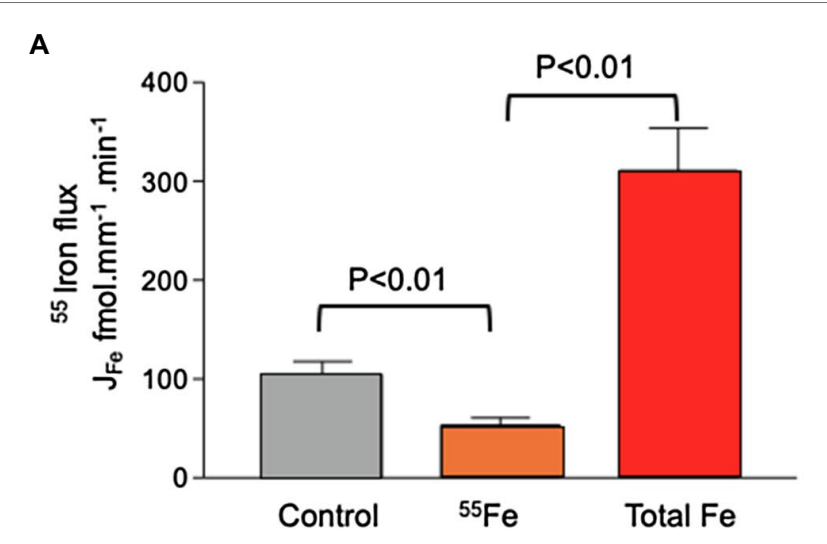

B

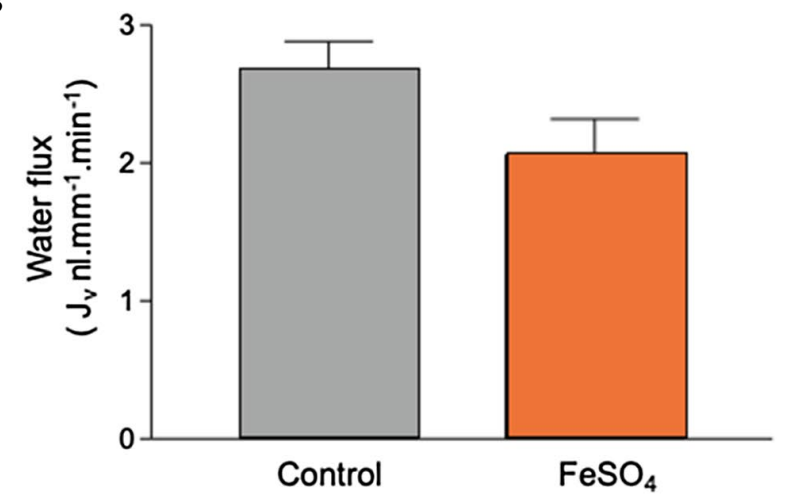

C

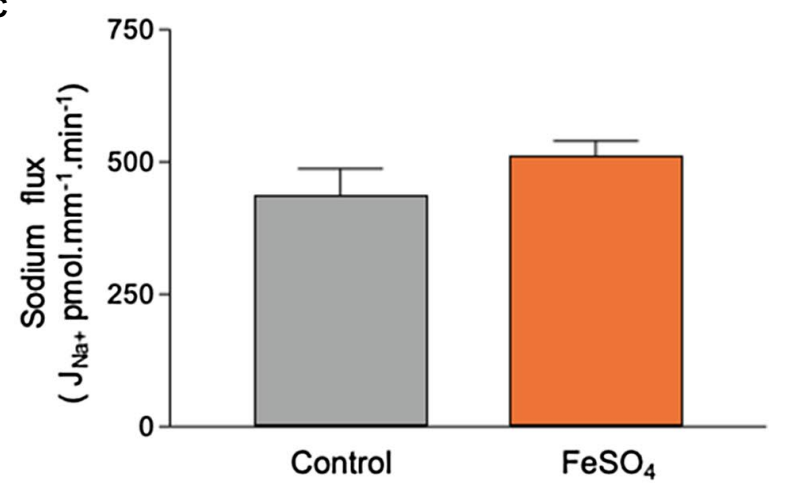

FIGURE 4 | Effect of $60 \mu$ mol. I. $^{-1} \mathrm{FeSO}_{4}$ on ${ }^{55} \mathrm{Fe}$, water, and $\mathrm{Na}^{+}$flux in microperfused renal PCTs. Surface S2 PCTs were microperfused in vivo. (A) Addition of $60 \mu \mathrm{mol}^{.} \mathrm{l}^{-1} \mathrm{FeSO}_{4}$ reduced ${ }^{55} \mathrm{Fe}$ flux from $105.2 \pm 12.7 \mathrm{fmol}$. $\mathrm{mm}^{-1} \cdot \mathrm{min}^{-1}$ to $53.5 \pm 7.5 \mathrm{fmol} \cdot \mathrm{mm}^{-1} \cdot \mathrm{min}^{-1}(n=11 ; p<0.01)$. Recalculating the molar activity of ${ }^{55} \mathrm{Fe}$ to take into account the addition of $60 \mu \mathrm{mol} . .^{-1}$ iron denoted "Total Fe" revealed that iron transport as a whole increased to $310.5 \pm 43.4 \mathrm{fmol} \cdot \mathrm{mm}^{-1} \cdot \mathrm{min}^{-1}(n=11 ; \mathrm{p}<0.01)$. (B) Mean water flux was reduced by inclusion of $60 \mu \mathrm{mol}^{. l^{-1}} \mathrm{FeSO}_{4}$ from $2.7 \pm 0.2$ to $2.1 \pm 0.3 \mathrm{nl} . \mathrm{mm}^{-1}$. $\min ^{-1}(n=11)$, but the observed reduction was not statistically significant at p,0.5 level. (C) Sodium flux by microperfused PCT $(428.6 \pm 57.2 ; n=19$. Addition of $60 \mu \mathrm{mol}^{.} \mathrm{I}^{-1} \mathrm{FeSO}_{4}$ gave a mean value of $514.80 \pm 28.6 \mathrm{nmol} . \mathrm{mm}^{-1}$. $\min ^{-1} ; n=11$.

by inclusion of ascorbic acid; therefore, the finding that ascorbate did not increase flux argues against involvement of DMT1, ZIP8, or ZIP14 in transporting Fe out of the
A

\section{Iron transport}

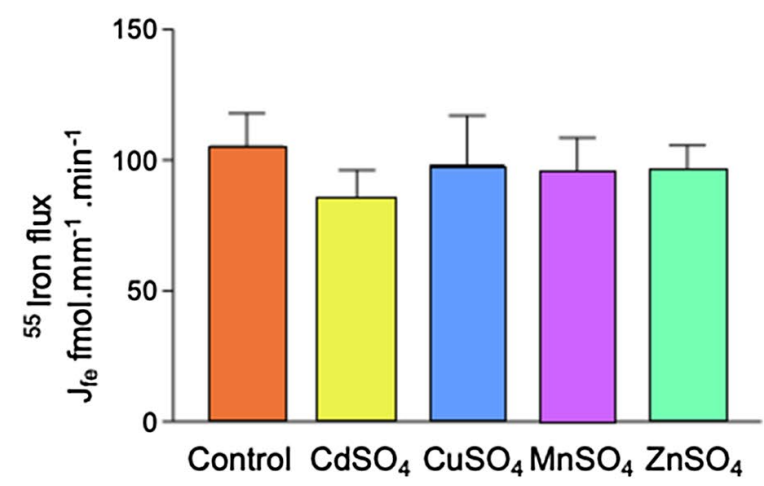

B

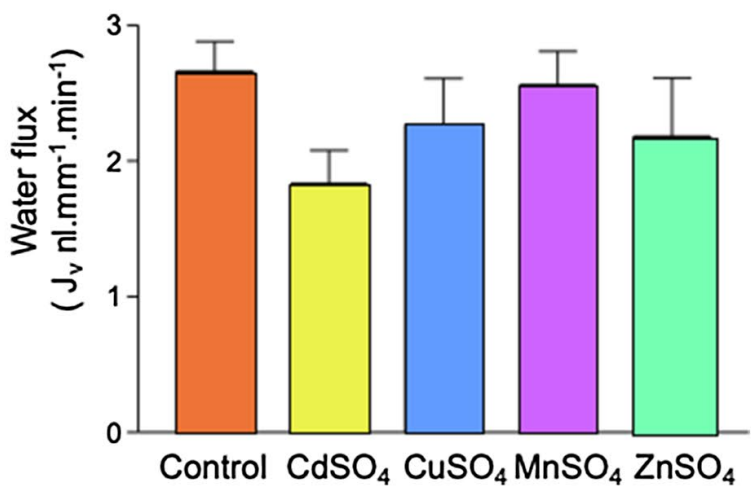

C

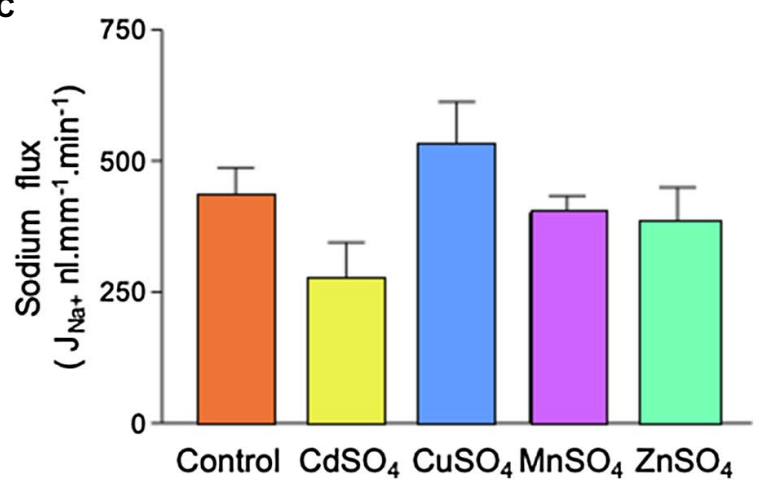

FIGURE 5 | Effect of competing divalent metal addition on ${ }^{55} \mathrm{Fe}$, water and $\mathrm{Na}^{+}$flux in microperfused renal PCTs. Tubules were perfused with $12 \mu \mathrm{mol} . .^{-1}$ ${ }^{55} \mathrm{FeCl}_{3}$ (control) and $\mathrm{CdSO}_{4}, \mathrm{CuSO}_{4}, \mathrm{MnSO}_{4}$, or $\mathrm{ZnSO}_{4}$ were included $\left(12 \mu \mathrm{mol}^{. \mathrm{I}^{-1}}\right)$. (A) ${ }^{55} \mathrm{Fe}$ flux was unaffected by competing metals. (B) Inclusion of competing metals had no statistically significant effect on water flux. (C) Inclusion of competing metals had no statistically significant effect on $\mathrm{Na}^{+}$ flux. Number of replicates were as follows: control $=19, \mathrm{CdSO}_{4}=9, \mathrm{CuSO}_{4}=9$ $\mathrm{MnSO}_{4}=8$, or $\mathrm{ZnSO}_{4}=7$.

PCT. Interestingly, ascorbate caused water flux to increase marginally. This might reflect the transport activity of ascorbate by the $\mathrm{Na}+-$ coupled L-ascorbic acid transporter SVCT1. SVCT1 is expressed in the PCT, and like SGLT1 is likely 
to transport water and its cognate substrate (Castro et al., 2008; Mackenzie et al., 2008; Bürzle et al., 2013).

To further probe the reabsorptive pathway, we performed ion competition experiments. A prominent characteristic of DMT1, ZIP8, and ZIP14 is that in addition to Fe, these proteins transport other divalent metals: DMT1 has substrate selectivity for $\mathrm{Cd}^{2+}, \mathrm{Co}^{2+}, \mathrm{Mn}^{2+}$, and to a lesser extent $\mathrm{Zn}^{2+} \mathrm{ZIP} 8$ has selectivity for $\mathrm{Cd}^{2+}, \mathrm{Co}^{2+}, \mathrm{Zn}^{2+}$, and to a lesser extent $\mathrm{Mn}^{2+}$ and ZIP 14 has the capacity to mediate transport of $\mathrm{Cd}^{2+}$, $\mathrm{Mn}^{2+}$, and $\mathrm{Zn}^{2+}$ (Sparkman et al., 2011; Illing et al., 2012; Wang et al., 2012). In light of this, we microperfused tubules with solutions containing $\mathrm{Cd}, \mathrm{Zn}, \mathrm{Cu}$, or $\mathrm{Mn}$. Addition of competing metals did not significantly alter $\mathrm{Fe}$, sodium, or water flux, although addition of $\mathrm{CdSO}_{4}$ to the perfusate caused a marginal decrease in sodium and water flux that did not reach statistical significance. Taken together, these data suggest DMT1, ZIP8, and ZIP14 are unlikely major contributors to unidirectional Fe flux out of the PCT.

In our previous work, we proposed that DMT1 is responsible for transport of $\mathrm{Fe}$ across the apical membrane of the late loop of Henle and early distal tubule because we showed DMT1 is expressed in the apical plasma membrane of these segments and transport of $\mathrm{Fe}$ was reduced by inclusion of other divalent metals which are known ligands of DMT (Wareing et al., 2000; Ferguson et al., 2001). In contrast, DMT1 is unlikely to transport Fe across the apical PCT membrane based on the functional studies presented in this work and this is supported by robust immunolabelling studies showing DMT1 expressed in late endosomes and lysosomes of PCT cells in rodents and not in the apical plasma membrane (Abouhamed et al., 2006).

If not DMT1, then could ZIP8 or ZIP14 be responsible for PCT Fe flux? In order to fulfil this role, these transporters would have to be expressed in the apical PCT membrane. Unfortunately, to date, it is not possible to discern with any degree of certainty whether these proteins are expressed in this membrane or like DMT1 whether they are expressed in a subcellular compartment. Furthermore, in the current study none of the competing divalent metals we trialed which are known ligands of ZIP8 or ZIP14 inhibited Fe transport indicating that in the absence of pathology, neither of these proteins mediate transport of $\mathrm{Fe}$ in the segments we investigated (mainly S2). In support of this tentative conclusion, ZIP8 has negligible transport activity at $\mathrm{pH} 6.5$, and the prevailing $\mathrm{pH}$ in the S2 segment is 2.7 (Dubose et al., 1979; Wang et al., 2012). In comparison, ZIP14 shows some activity at pH6.5 (Sparkman et al., 2011); however, the lack of effect of zinc on Fe transport argues strongly against the proposed involvement of this protein in PCT apical membrane Fe flux (Liuzzi et al., 2006; Fujishiro et al., 2012).

Other potential candidate mechanisms are apparent in the PCT. The megalin/cubilin receptor complex and TFR1 are both expressed in the apical PCT membrane are likely to be responsible for reabsorbing filtered transferrin bound Fe (Christensen et al., 2012; Smith et al., 2019). Our micropuncture experiments simply demonstrated that $\mathrm{Fe}$ was present in ultrafiltrate and that the $\mathrm{Fe}$ concentration profile along the proximal tubule strongly indicated reabsorption; however, we did not assess what species of Fe was present. The tubular perfusion experiments we performed used a perfusate which was protein-free and contained $\mathrm{Fe}$ in its unbound state - sometimes referred to as non-transferrin bound $\mathrm{Fe}$. We specifically designed the experiments to enable us to functionally test for the presence of DMT1, ZIP8, and ZIP14 since these transporters translocate unbound $\mathrm{Fe}$. Clearly, future experiments should aim to examine the role of receptor-mediated mechanisms while bearing in mind the observations of the current study that indicate the presence of transport of elemental unbound- Fe.

Overall based on the data presented in this work, what is the contribution of renal $\mathrm{Fe}$ reabsorption to $\mathrm{Fe}$ balance? We propose that in addition to its role as a source of EPO, the kidney is critical for maintaining Fe balance due to the following reasoning: Within serum, the concentration of Fe measured using the TCA precipitation method was $50.5 \pm 3.9 \mu \mathrm{mol}^{-1} \mathrm{I}^{-1}$. The majority of this represents Fe bound to transferrin, and some of this passes the glomerular filtration barrier and becomes a component of the ultrafiltrate. The plasma volume of a $250 \mathrm{~g}$ rat is $9.8 \mathrm{mls}$ (Bijsterbosch et al., 1981); therefore, the total amount of Fe in plasma is $0.49 \mu \mathrm{mol}$. In vitro we measured the ultrafiltered concentration of Fe to be $4.5 \mu \mathrm{mol} . \mathrm{I}^{-1}$ and in vivo the mean PCT concentration in the S2 segment to be $3.0 \mu \mathrm{mol}^{-\mathrm{l}^{-1}}$. Assuming a mean glomerular filtration rate of a $250 \mathrm{~g}$ rat is $2.251 . \mathrm{day}^{-1}$, then between 6.9 and $10.1 \mu \mathrm{mol} \mathrm{Fe}$ would be filtered per day. In terms of maintaining $\mathrm{Fe}$ balance, the implication of this estimate is that the total amount of $\mathrm{Fe}$ in plasma would be lost in less than two hours if the kidney did not reabsorb filtered Fe. From the available data, we constructed a model of renal $\mathrm{Fe}$ handling (see Figure 6). It can be seen that the bulk of $\mathrm{Fe}$ is reabsorbed in the PCT, by a non-DMT1-dependent pathway that is not shared by copper, manganese, or zinc. There is significant DMT1-mediated transport of $\mathrm{Fe}$ by the loop of Henle and the collecting duct system which in comparison may also mediate transport of copper and manganese and to some extent zinc.

Micropuncture is not possible in human subjects, and imaging methods are not sufficiently refined to resolve nephron distribution of Fe; however, recent data suggest that transporter proteins necessary to affect epithelial $\mathrm{Fe}$ transport are present in the human kidney and may in some cases be implicated in deleterious renal Fe accumulation (Van Raaij et al., 2018). And although it can be inferred that $\mathrm{Fe}$ is filtered by human kidneys, definitive localization studies to pinpoint the nephron expression of transporter proteins are lacking and should ideally be the focus of future studies.

In conclusion, we have demonstrated that $\mathrm{Fe}$ is present in ultrafiltrate in the S2 PCT. The Fe concentration profile along the PCT is indicative of PCT Fe reabsorption. The mechanism responsible for the observed flux remains obscure; however, DMT1, ZIP8, or ZIP14 are unlikely to majorly contribute. We propose that the rat kidney filters and reabsorbs $\mathrm{Fe}$ and this process is potentially of critical importance to Fe homeostasis. 




FIGURE 6 | Renal iron transport. Model of renal iron handling along the nephron showing estimated quantitative contribution of three groups of nephron segments. Percentage data refer to the amount of filtered iron reabsorbed. Values apply to a $250 \mathrm{~g}$ Wistar rat and are based on data presented in this manuscript and Wareing et al., 2000). Filtered load of iron was calculated assuming a glomerular filtration rate of 2.25l.day ${ }^{-1}$ (Bijsterbosch et al., 1981) and taking the $30 \mathrm{kDa}$ ultrafiltrate iron concentration of $4.48 \mu \mathrm{mol}^{. I^{-1}}$ as representative of the concentration of iron filtered. Iron remaining at the end of the PCT was calculated assuming $60 \%$ of filtered water is reabsorbed along PCT and taking the end PCT iron concentration to be $1.8 \mu$ mol..$^{-1}$ (from Figure 1). PCT reabsorption of iron was calculated by subtracting end PCT iron concentration from the filtered load of iron. The amount of iron reabsorbed by the loop of Henle or DCT and collecting ducts was calculated using the urinary iron concentration as a starting point (value taken Wareing et al., 2000,) and assuming $20 \%$ of injected iron was reabsorbed downstream of the PCT (value taken Wareing et al., 2000). Iron present at the start of the DCT was calculated assuming 50\% of injected iron was reabsorbed downstream of the DCT. Iron reabsorbed by loop of Henle was calculated by subtracting the calculated value for the DCT from the measured iron concentration at the end of the PCT (from Figure 1).

\section{DATA AVAILABILITY STATEMENT}

The original contributions presented in the study are included in the article/supplementary material, and further inquiries can be directed to the corresponding author.

\section{AUTHOR CONTRIBUTIONS}

All authors listed have made a substantial, direct and intellectual contribution to the work, and approved it for publication.

\section{REFERENCES}

Abouhamed, M., Gburek, J., Liu, W., Torchalski, B., Wilhelm, A., Wolff, N. A., et al. (2006). Divalent metal transporter 1 in the kidney proximal tubule is expressed in late endosomes/lysosomal membranes: implications for renal handling of protein-metal complexes. Am. J. Physiol. - Ren. Physiol. 290, 1525-1533. doi: 10.1152/ajprenal.00359.2005

\section{FUNDING}

We gratefully acknowledge the financial support of the Wellcome Trust Grant no. 043322/Z/94 (CPS) and the Royal Society (CPS).

\section{ACKNOWLEDGMENTS}

The authors would like to thank Prof. Roger Green for his guidance and insight.

Ajjimaporn, A., Botsford, T., Garrett, S. H., Sens, M. A., Zhou, X. D., Dunlevy, J. R., et al. (2012). ZIP8 expression in human proximal tubule cells, human urothelial cells transformed by $\mathrm{cd}+2$ and $\mathrm{As}+3$ and in specimens of normal human urothelium and urothelial cancer. Cancer Cell Int. 12, 1-14. doi: $10.1186 / 1475-2867-12-16$

Bank, N., and Aynedjian, H. S. (1972). Techniques of microperfusion of renal tubules and capillaries. Yale J. Biol. Med. 45, 312-317. 
Bijsterbosch, M. K., Duursma, A. M., Bouma, J. M. W., and Gruber, M. (1981). The plasma volume of the Wistar rat in relation to the body weight. Experientia 37, 381-382. doi: 10.1007/BF01959874

Bürzle, M., Suzuki, Y., Ackermann, D., Miyazaki, H., Maeda, N., Clémençon, B., et al. (2013). The sodium-dependent ascorbic acid transporter family SLC23. Mol. Asp. Med. 34, 436-454. doi: 10.1016/j.mam.2012.12.002

Castro, T., Low, M., Salazar, K., Montecinos, H., Cifuentes, M., Yáñez, A. J., et al. (2008). Differential distribution of the sodium-vitamin C cotransporter-1 along the proximal tubule of the mouse and human kidney. Kidney Int. 74, 1278-1286. doi: 10.1038/ki.2008.329

Christensen, E. I., Birn, H., Storm, T., Weyer, K., and Nielsen, R. (2012). Endocytic receptors in the renal proximal tubule. Physiology 27, 223-236. doi: $10.1152 /$ physiol.00022.2012

Dubose, T. D., Pucacco, L. E. O. R., Seldin, D. W., Carter, N. W., and Kokko, J. P. (1979). Microelectrode determination of $\mathrm{pH}$ and $\mathrm{PCO} 2$ in rat proximal tubule after benzolamide: evidence for hydrogen ion secretion. Kidney Int. 15, 624-629. doi: 10.1038/ki.1979.82

Ferguson, C. J., Wareing, M., Ward, D. T., Green, R., Smith, C. P., and Riccardi, D. (2001). Cellular localization of divalent metal transporter DMT-1 in rat kidney. Am. J. Physiol. - Ren. Physiol. 280, F803-F814. doi: 10.1152/ ajprenal.2001.280.5.F803

Fernandez, F., and Kahn, H. (1971). Clinical methods for atomic absorption spectroscopy. Clin. Chem. News. 3:24

Fujishiro, H., Yano, Y., Takada, Y., Tanihara, M., and Himeno, S. (2012). Roles of ZIP8, ZIP14, and DMT1 in transport of cadmium and manganese in mouse kidney proximal tubule cells. Metallomics 4:700. doi: 10.1039/c2mt20024d

Green, R., Windhager, E. E., and Giebisch, G. (1974). Protein oncotic pressure effects on proximal tubular fluid movement in the rat. Am. J. Phys. 226, 265-276. doi: 10.1152/ajplegacy.1974.226.2.265

Gunshin, H., Mackenzie, B., Berger, U. V., Gunshin, Y., Romero, M. F., Boron, W. F., et al. (1997). Cloning and characterization of a mammalian proton-coupled metal-ion transporter. Nature 388, 482-488. doi: 10.1038/ 41343

Illing, A. C., Shawki, A., Cunningham, C. L., and Mackenzie, B. (2012). Substrate profile and metal-ion selectivity of human divalent metal-ion transporter-1. J. Biol. Chem. 287, 30485-30496. doi: 10.1074/jbc.M112.364208

Kozyraki, R., Fyfe, J., Verroust, P. J., Jacobsen, C., Dautry-Varsat, A., Gburek, J., et al. (2001). Megalin-dependent cubilin-mediated endocytosis is a major pathway for the apical uptake of transferrin in polarized epithelia. Proc. Natl. Acad. Sci. U. S. A. 98, 12491-12496. doi: 10.1073/pnas.211291398

Liuzzi, J. P., Aydemir, F., Nam, H., Knutson, M. D., and Cousins, R. J. (2006). Zip14 (Slc39a14) mediates non-transferrin-bound iron uptake into cells. Proc. Natl. Acad. Sci. 103, 13612-13617. doi: 10.1073/pnas.0606424103

Mackenzie, B., Illing, A. C., and Hediger, M. A. (2008). Transport model of the human $\mathrm{Na}+-$ coupled L-ascorbic acid (vitamin C) transporter SVCT1. Am. J. Phys. Cell Phys. 294, 451-459. doi: 10.1152/ajpcell.00439.2007

Olson, A. D., and Hamlin, W. B. (1969). A new method for serum iron and total iron-binding capacity by atomic absorption spectrophotometry. Clin. Chem. 15, 438-444. doi: 10.1093/clinchem/15.6.438

Ponka, P. (1999). Cellular iron metabolism. Kidney Int. Suppl. 69, S2-S11. doi: 10.1046/j.1523-1755.1999.055suppl.69002.x

Rector, F. C. J. (1983). Sodium, bicarbonate, and chloride absorption by the proximal tubule. Am. J. Phys. 244, F461-F471. doi: 10.1152/ajprenal.1983. 244.5.F461

Shalmi, M., Kibble, J. D., Day, J. P., Christensen, P., and Atherton, J. C. (1994). Improved analysis of picomole quantities of lithium, sodium, and potassium in biological fluids. Am. J. Phys. 267, F695-F701. doi: 10.1152/ ajprenal.1994.267.4.F695
Smith, C. P., Lee, W.-K., Haley, M., Poulsen, S. B., Thevenod, F., and Fenton, R. A. (2019). Proximal tubule transferrin uptake is modulated by cellular iron and mediated by apical membrane megalin-cubilin complex and transferrin receptor 1. J. Biol. Chem. 294, 7025-7036. doi: 10.1074/jbc.RA118.006390

Smith, C. P., and Thévenod, F. (2009). Iron transport and the kidney. Biochim. Biophys. Acta, Gen. Subj. 1790, 724-730. doi: 10.1016/j.bbagen.2008.10.010

Sparkman, B. K., Illing, A. C., Mackenzie, B., Liuzzi, J. P., Mitchell, C. J., Knutson, M. D., et al. (2011). Zip14 is a complex broad-scope metal-ion transporter whose functional properties support roles in the cellular uptake of zinc and nontransferrin-bound iron. Am. J. Physiol. Physiol. 301, C862C871. doi: 10.1152/ajpcell.00479.2010

Thévenod, F., and Wolff, N. A. (2016). Iron transport in the kidney: implications for physiology and cadmium nephrotoxicity. Metallomics 8, 17-42. doi: 10.1039/C5MT00215J

Raaij, S.Van, Swelm, R.Van, Bouman, K., Cliteur, M., Den, M. C.Van, Pertijs, J., et al. (2018). Tubular iron deposition and iron handling proteins in human healthy kidney and chronic kidney disease. Sci. Rep. 8:9353. doi:10.1038/s41598-018-27107-8.

Wang, C. Y., Jenkitkasemwong, S., Duarte, S., Sparkman, B. K., Shawki, A., Mackenzie, B., et al. (2012). ZIP8 is an iron and zinc transporter whose cell-surface expression is up-regulated by cellular iron loading. J. Biol. Chem. 287, 34032-34043. doi: 10.1074/jbc.M112.367284

Wareing, M., Ferguson, C. J., Green, R., Riccardi, D., and Smith, C. P. (2000). In vivo characterization of renal iron transport in the anaesthetized rat. J. Physiol. 524, 581-586. doi: 10.1111/j.1469-7793.2000.00581.x

Wareing, M., and Green, R. (1994). Effect of formate and oxalate on fluid reabsorption from the proximal convoluted tubule of the anaesthetized rat. J. Physiol. 477, 347-354. doi: 10.1113/jphysiol.1994.sp020196

Wareing, M., Wilson, R. W., Kibble, J. D., and Green, R. (1995). Estimated potassium reflection coefficient in perfused proximal convoluted tubules of the anaesthetized rat in vivo. J. Physiol. 488, 153-161. doi: 10.1113/jphysiol.1995. sp020953

Williams, R. J. P. (2012). Iron in evolution. FEBS Lett. 586, 479-484. doi: 10.1016/j.febslet.2011.05.068

Wilson, R. W., Wareing, M., and Green, R. (1997). The role of active transport in potassium reabsorption in the proximal convoluted tubule of the anaesthetized rat. J. Physiol. 500, 155-164. doi: 10.1113/jphysiol.1997.sp022006 Wolff, N. A., Liu, W., Fenton, R. A., Lee, W.-K., Thévenod, F., and Smith, C. P. (2011). Ferroportin 1 is expressed basolaterally in rat kidney proximal tubule cells and iron excess increases its membrane trafficking. J. Cell. Mol. Med. 15, 209-219. doi: 10.1111/j.1582-4934.2009.00985.x

Conflict of Interest: The authors declare that the research was conducted in the absence of any commercial or financial relationships that could be construed as a potential conflict of interest.

Publisher's Note: All claims expressed in this article are solely those of the authors and do not necessarily represent those of their affiliated organizations, or those of the publisher, the editors and the reviewers. Any product that may be evaluated in this article, or claim that may be made by its manufacturer, is not guaranteed or endorsed by the publisher.

Copyright () 2021 Wareing and Smith. This is an open-access article distributed under the terms of the Creative Commons Attribution License (CC BY). The use, distribution or reproduction in other forums is permitted, provided the original author(s) and the copyright owner(s) are credited and that the original publication in this journal is cited, in accordance with accepted academic practice. No use, distribution or reproduction is permitted which does not comply with these terms. 